\title{
Closed-loop controller for post-impact vehicle dynamics using individual wheel braking and front axle steering
}

\section{Derong Yang* and Bengt Jacobson}

Department of Applied Mechanics, Chalmers University of Technology, SE-41296 Gothenburg, Sweden Email: derong.yang@volvocars.com Email: bengt.jacobson@chalmers.se *Corresponding author

\section{Mats Jonasson}

Department of Vehicle Dynamics and Active Safety, Volvo Car Corporation, SE-40531 Gothenburg, Sweden

Email: mats.jonasson@volvocars.com

\author{
Tim J. Gordon \\ Department of Mechanical Engineering, \\ University of Michigan, \\ Ann Arbor, MI 48109, USA \\ Email: tjgordon@umich.edu
}

\begin{abstract}
This paper presents a vehicle path controller for reducing the maximum lateral deviation $\left(Y_{\max }\right)$ after an initial impact in a traffic accident. In previous research, a Quasi-Linear Optimal Controller (QLOC) was proposed and applied to a simple vehicle model with individually controlled brake actuators. QLOC uses non-linear optimal control theory to provide a semiexplicit approximation for optimal post-impact path control, and in principle can be applied to an arbitrary number of actuators. The current work extends and further validates the control method by analysing the effects of adding an active front axle steering actuator at different post-impact kinematics, as well as increasing the fidelity of the vehicle model in the closed-loop controlled system. The controller performance is compared with the results from openloop numerical optimisation which uses the same vehicle model. The inherent robustness properties of the QLOC algorithm are demonstrated by its direct application to an independent high-fidelity multi-body vehicle model. Towards real-time implementation, the algorithm is further simplified so that the computational efficiency is enhanced, whereas the performance is shown not to be degraded.
\end{abstract}

Keywords: collision avoidance; active safety; post-impact; path control; vehicle dynamics; braking; steering; optimal control. 
Reference to this paper should be made as follows: Yang, D., Jacobson, B., Jonasson, M. and Gordon, T.J. (2014) 'Closed-loop controller for post-impact vehicle dynamics using individual wheel braking and front axle steering', Int. J. Vehicle Autonomous Systems, Vol. 12, No. 2, pp.158-179.

Biographical notes: Derong Yang is currently working towards the $\mathrm{PhD}$ degree in Vehicle and Machine Systems, at the Vehicle Dynamics Research Group, Department of Applied Mechanics at Chalmers University of Technology, Gothenburg, Sweden. She received the BSc degree in Vehicle Engineering from Wuhan University of Technology, Wuhan, China, in 2007 and the MSc degree in Automotive Engineering, in 2009, from Chalmers University of Technology. Her research interests include the vehicle dynamics and control in crash-imminent scenarios, for instance the post-impact vehicle motion control for mitigation of secondary events in multiple-event accidents.

Bengt Jacobson is currently a Professor of Vehicle Dynamics with the Department of Applied Mechanics, Chalmers University of Technology. The focus of this research group is vehicle motion in the ground plane for both passenger cars and heavy trucks. He received the $\mathrm{PhD}$ degree in Machine Elements from Chalmers University of Technology, Gothenburg, Sweden, in 1993, studying the dynamics of gear shifting in power-shifting transmissions. He later continued research on longitudinal vehicle dynamics with dynamic modelling and control of power train systems, including hybrid vehicles. Based on this topic and teaching in vehicle dynamics, he was entitled as Associate Professor in 1998. He was a Technical Expert (2001-2010) with Volvo Car Corporation, Gothenburg, with focus on vehicle dynamics control and active safety, mainly brakes and steering.

Mats Jonasson is currently a Functional Developer with the Department of Vehicle Dynamics Functions, Volvo Car Corporation, working in the field of vehicle dynamics control. In addition, he is an affiliated researcher at KTH Vehicle Dynamics. He received the MSc degree in Electrical Engineering from Chalmers University of Technology, Gothenburg, Sweden, and $\mathrm{PhD}$ degree in Vehicle Engineering from Royal Institute of Technology (KTH), Stockholm, Sweden, in 2009, exploiting individual wheel actuators to enhance vehicle dynamics and safety in electric vehicles. Since 1998, he has been with Volvo Car Corporation, Gothenburg. He has experience in design engineering.

Tim J. Gordon is currently a Research Professor with the Department of Mechanical Engineering, University of Michigan, Ann Arbor. He was previously the Department Head for Aeronautical and Automotive Engineering, Loughborough University, Leicestershire, UK, where he held the Ford Chair in Automotive Systems Engineering. He also co-chairs an IEEE Technical Committee (Intelligent Vehicular Systems and Control) and is the Chair of a standing committee for the Transportation Research Board of the National Academies (Vehicle-Highway Automation). He received the BA and MA degrees in Mathematics from Cambridge University, Cambridge, UK, in 1974 and 1975, respectively, and the PhD degree in Applied Mathematics from the Department of Applied Mathematics and Theoretical Physics, Cambridge University, in 1983.

This paper is a revised and expanded version of a paper entitled 'Closed-loop controller for post-impact vehicle dynamics using individual wheel braking and front axle steering' presented at the 'International Conference on Advance Vehicle Technologies and Integration', ChangChun, China, 16-19 July 2012. 


\section{Introduction}

Accident statistics show that Multiple-Event Accidents (MEAs) constitute an increasing fraction of all accidents (Sander et al., 2009; Zhou et al., 2010). MEAs are characterised by having at least one vehicle subjected to more than one harmful event, such as collision with another vehicle. MEAs now comprise up to a third of all passenger vehicle accidents; human injury levels in most of MEAs are higher than in single-event accidents due to the subsequent events after the first impact (Yang et al., 2009; Fay et al., 2001).

Previous research has investigated the capability of minimising the maximum path lateral deviation $Y_{\max }$ from the original path (Yang et al., 2011), and a non-linear path controller was proposed using individual wheel braking (Yang et al., 2012b). There the closed-loop controller incorporates three sub-strategies of the optimal brake strategy identified at different severity levels of post-impact states: (a) at large yaw velocities, lateral force control is dominant, where the lateral force is instantaneously maximised in the desired global direction of path recovery; (b) at moderate yaw velocities, coupled force-moment control is required to balance the global lateral force vector versus the stabilising yaw moment; a Quasi-Linear Optimal Controller (QLOC) was proposed to determine this balance optimally (Yang et al., 2012a); and (c) after the point of maximum path deviation, an approximately linear settling stage allows the vehicle to recover towards the original path.

The present work aims to extend the domain of the QLOC controller by adding a front axle steering actuator, where it is expected that performance with respect to $Y_{\max }$ reduction can be largely improved, as shown in the previous open-loop optimisations (Yang et al., 2012c). Active steering control may not always be a realistic option, as this concerns the choice of action by the human driver. Accident statistics and driving tests have partly shown that the driver hardly corrects or applies control to the steering in the presence of large disturbances, as following an external impact to the car body (Häussler et al., 2012; Kusachov and Mouatamid, 2012). However, it is also possible that the driver would act to control the steering angle himself/herself. These issues about driver overriding are not considered in the controller design here, i.e. autonomous vehicle actions are assumed for some seconds immediately after the first impact.

The rest of the paper is structured as follows. The vehicle model for both controller design and numerical optimisation is introduced in Section 2. Using the reduced version of the vehicle model, in Section 3 the closed-loop control is built based on non-linear optimal control theory. Section 4 presents two specific cases and the results from offline numerical optimisation are used as an independent test of optimality. In favour of realtime experiments, the algorithm is simplified by considering the actuators limits in Section 5. In Section 6, the proposed controller is further tested on another vehicle model which has rather more degrees of freedom and was validated for limit handling manoeuvres. Section 7 discusses about future work and concludes the paper.

\section{Vehicle model and reduced-order system equations}

\subsection{Vehicle model}

The vehicle model used in both closed-loop simulation and open-loop optimisation is a 6DOF vehicle model with three translational velocities (longitudinal, lateral and vertical) 
and three rotational velocities (yaw, roll and pitch), relative to the vehicle coordinates. The equations of motion are shown in equation (1), using notations given in the Appendix A:

$$
\begin{aligned}
& m\left(\dot{v}_{x}-v_{y} \dot{\psi}+\ddot{\theta} h_{p}\right)=\left(F_{x 1}+F_{x 2}\right) \cos \delta_{f}+F_{x 3}+F_{x 4}-\left(F_{y 1}+F_{y 2}\right) \sin \delta_{f} \\
& m\left(\dot{v}_{y}+v_{x} \dot{\psi}-\ddot{\phi} h_{r}\right)=\left(F_{y 1}+F_{y 2}\right) \cos \delta_{f}+F_{y 3}+F_{y 4}+\left(F_{x 1}+F_{x 2}\right) \sin \delta_{f} \\
& m\left(\dot{v}_{z}+g\right)=F_{z 1}+F_{z 2}+F_{z 3}+F_{z 4} \\
& I_{x x} \ddot{\phi}-m_{r}\left(\dot{v}_{y}+v_{x} \dot{\psi}-\ddot{\phi} h_{r}\right)=\frac{w}{2}\left(F_{z 1}-F_{z 2}+F_{z 3}-F_{z 4}\right) \\
& I_{y y} \ddot{\theta}+m h_{p}\left(\dot{v}_{x}-v_{y} \dot{\psi}+\ddot{\theta} h_{p}\right)=-l_{f}\left(F_{z 1}+F_{z 2}\right)+l_{r}\left(F_{z 3}+F_{z 4}\right) \\
& I_{z z} \ddot{\psi}=l_{f}\left(F_{y 1}+F_{y 2}\right) \cos \delta_{f}-l_{r}\left(F_{y 3}+F_{y 4}\right)+l_{f}\left(F_{x 1}+F_{x 2}\right) \sin \delta_{f} \\
& +\frac{w}{2}\left(-F_{x 1}+F_{x 2}\right) \cos \delta_{f}+\frac{w}{2}\left(-F_{x 3}+F_{x 4}\right)+\frac{w}{2}\left(F_{y 1}-F_{y 2}\right) \sin \delta_{f}
\end{aligned}
$$

Note that the products of inertia are neglected. The vertical forces are calculated at each wheel according to the suspension vertical displacements and velocities. The actuator dynamics, i.e. limits of Friction Brakes (FricBrk) and Electric Power Assist Steer (EPAS) systems, are also included in the vehicle model. Parameters of a sedan-type vehicle are adopted according to the vehicle data used in Yang et al. (2011) and Sundström et al. (2010). A simplified version of the Pacejka (2006) tyre model is used, with longitudinal force $F_{x i}$ as input variable, which is limited by the available road-tyre friction, the current vertical loads and tyre side-slip angles (see equations 2 and 3). Here $D_{i}$ is the peak lateral tyre force formulated as a function of tyre longitudinal force $F_{x i}$, friction coefficient $\mu_{i}$ and vertical load $F_{z i}$. The tyre relaxation effect is modelled as a first-order lag (see equation 4). Vehicle parameters are given in Appendix A:

$$
\begin{aligned}
& F_{y i}\left(\alpha_{i}\right)=-D_{i} \cdot \sin \left(C_{i} \cdot \arctan \left(B_{i} \cdot \alpha_{i}\right)\right) \\
& D_{i}=\sqrt{\left(\mu_{i} \cdot F_{z i}\right)^{2}-F_{x i}^{2}}
\end{aligned}
$$

where

$$
F_{x i}= \begin{cases}-\operatorname{sgn}\left(v_{x w i}\right) \cdot \mu_{i} F_{z i} \cos \alpha_{i}, & \left|F_{x w i}\right| \geq \mu_{i} F_{z i} \cos \alpha_{i} \\ -\operatorname{sgn}\left(v_{x w i}\right) \cdot\left|F_{x w i}\right|, & \left|F_{x w i}\right|<\mu_{i} F_{z i} \cos \alpha\end{cases}
$$

and

$$
\dot{\alpha}_{i}=\frac{v_{x i}}{L_{y i}} \cdot\left[-\alpha_{i}-\delta_{i}+\arctan \left(\frac{v_{y i}}{v_{x i}}\right)\right]
$$

The controlled system state equations can hereby be written in a general form:

$$
\begin{aligned}
& \dot{\mathbf{x}}=\mathbf{f}(\mathbf{x}(t), \mathbf{u}(t)), \quad \mathbf{x}(0)=\mathbf{x}_{0} \\
& y=p(\mathbf{x}(t))
\end{aligned}
$$

where $\mathbf{x}=\left[v_{x}, v_{y}, v_{z}, \dot{\psi}, \dot{\phi}, \dot{\theta}\right]^{\mathrm{T}}$ is the state vector and $\mathbf{u}=\left[F_{w 1}, F_{w 2}, F_{w 3}, F_{w 4}, \delta_{f}\right]^{\mathrm{T}}$ is the control input vector including the equivalent forces of the frictional brake torque at the brake discs, i.e. $F_{w i}$, and the steering angle $\delta_{f}$ at front axle, assuming equal left and right 
steer angles. The control output $y=Y(t)$ is the path lateral deviation, and $\mathbf{x}_{0}$ defines the post-impact initial states. Here vehicle parameters and tyre-ground contact adhesion coefficient are assumed to be constant over time; thus, the model ( $f$ and $p$ ) is shown as time-invariant.

\subsection{Reduced model for controller design}

As stated in the Introduction, the controller aims to limit the path deviation in the road coordinate system; thus, for the purpose of controller design, we transform the velocity variables into global components in the road coordinates: $\tilde{\mathbf{x}}=[X, \dot{X}, Y, \dot{Y}, Z, \dot{Z}, \psi, \dot{\psi}, \phi, \dot{\phi}$, $\theta, \dot{\theta}]^{\mathrm{T}}$. We also transform from actuator inputs $\mathbf{u}$ to the resultant vehicle body forces and yaw moments: $\tilde{\mathbf{u}}=\left[\frac{F_{x g}^{a c t}}{m}, \frac{F_{y g}^{a c t}}{m}, \frac{F_{z g}^{a c t}}{m}, \frac{M_{z}^{a c t}}{I_{z z}}, \frac{M_{x}^{a c t}}{I_{x x}}, \frac{M_{y}^{a c t}}{I_{y y}}\right]^{\mathrm{T}}$. Here act denotes that we consider the active contributions from braking torques and steering angle inputs relative to their passive values, i.e. relative to $\mathbf{u}=0$. Each braking torque is transformed to the braking force at the corresponding tyre-ground contact patch $F_{x w i}$ which determines $F_{x i}$ and $F_{y i}$ via equations (2) and (3) and hence $\tilde{\mathbf{u}}$ is found. The dynamical system equations (5) can now be written as

$$
\begin{aligned}
& \dot{\tilde{\mathbf{x}}}=\mathbf{f}_{0}(\tilde{\mathbf{x}}(t))+B \cdot \tilde{\mathbf{u}}(t) \\
& y=C \cdot \tilde{\mathbf{x}}
\end{aligned}
$$

In the following, for simplicity, $\tilde{\mathbf{x}}$ is directly annotated as $\mathbf{x}$. Firstly, the abovementioned 12-state model is reduced to six states: $\mathbf{x}=[X, \dot{X}, Y, \dot{Y}, \psi, \dot{\psi}]^{\mathrm{T}}$. This is based on the assumption that the out-of-plane dynamics exert little overall bias on the vehicle dynamics within the road-plane. Thereafter, the six-state model is further reduced to four states: $\mathbf{x}=[Y, \dot{Y}, \psi, \dot{\psi}]^{\mathrm{T}}$, based on the observation that $X$-dynamics have little effect on the tyre side-slip angles and thus little effect on the forces and moments influencing lateral dynamics. Since the control objective does not depend explicitly on $X$, with this assumption the $Y$ and $\psi$ dynamics are decoupled from that of $X$. Correspondingly, the number of vehicle-level controls $\tilde{\mathbf{u}}$ is reduced to two: $\tilde{\mathbf{u}}=\left[\frac{F_{y g}^{a c t}}{m}, \frac{M_{z}^{a c t}}{I_{z z}}\right]^{\mathrm{T}}$. The dimensions of matrices $B$ and $C$ are likewise reduced.

The model is made linear by assuming small yaw angles $\psi(t)$ and constant longitudinal velocity $v_{x}$. The non-linear part $\mathbf{f}_{0}$ is linearised with underlying linear tyre characteristics. Here we assume an average value of the total cornering stiffness at each axle, i.e. $C_{\alpha_{f}}$ and $C_{\alpha_{r}}$, defined in the range of sustained tyre side-slip angles (instead of the linear cornering stiffness around zero side-slip). In the presence of active steering controls, the operating range of side-slip angles is pushed further into the non-linear region on tyre characteristic curve, as compared to values experienced for brake-only QLOC. Therefore, $C_{\alpha_{f}}$ and $C_{\alpha_{r}}$ are reduced, here by a factor of $1 / 4$, as compared to the 
values used for brake-only QLOC presented in Yang et al. (2012a). The state-space model in equation (6) is hereby simplified in equation (7) (see Yang et al., 2012a, for the complete form of the matrices A, B and C):

$$
\begin{aligned}
\dot{\mathbf{x}} & =A \cdot \mathbf{x}+B \cdot \tilde{\mathbf{u}} \\
y & =C \cdot \mathbf{x}
\end{aligned}
$$

\section{Closed-loop controller}

A QLOC was proposed which uses non-linear optimal control theory to provide a semiexplicit approximation for optimal post-impact path control (Yang et al., 2012a). The controller is defined as quasi-linear here in the way that it combines the linear co-states dynamics and the non-linear constraints due to tyre friction limits. The cost function $J$ is kept the same as compared to the previous work in Yang et al. (2012a), i.e. to minimise the maximum lateral deviation:

$$
J=\frac{1}{2} y_{f}^{2}=\frac{1}{2} Y_{f}^{2}=\frac{1}{2} Y_{\max }^{2}
$$

According to Pontryagin's minimum principle (Bryson and Ho, 1975), minimisation of the cost $J$ requires the scalar Hamiltonian function $H$ to be minimised for each time instant $t \in\left[0, t_{f}\right]$, where $t_{f}$ is the final time instant, when $\dot{Y}=0$ and thus $Y=Y_{\max }$.

The model in equation (7) is subjected to the above-mentioned control constraints on $\tilde{\mathbf{u}}$, since friction limits are respected at each individual wheel. After linearisation, the Hamiltonian can be written as

$$
H=\lambda^{\mathrm{T}} \cdot(A \mathbf{x}+B \tilde{\mathbf{u}})
$$

where the part influenced by the control inputs is $H_{1}=\lambda_{2} \tilde{u}_{1}+\lambda_{4} \tilde{u}_{2}$.

Since $\tilde{\mathbf{u}}$ appears linearly in the transformed state equations, the optimal control is fully determined by the active forces and moment constraints of the system:

$$
\min (H) \Leftrightarrow \min _{\tilde{\mathbf{u}}(t)}\left(\lambda^{\mathrm{T}}(t) \tilde{\mathbf{u}}(t)\right)
$$

The reduced model in Section 2.2 makes it possible to find an easily computed solution to the co-states dynamics. Constrained minimisation of $H_{1}$ is illustrated in Figure 1 . The cyan shaded region $U$ represents the bounded set of forces and moments available as the brake forces and front axle steering angle are varied; this is computed by a so-called 'brute-force' method (Jonasson et al., 2010). In Figure 1, the minimum value is achieved on the boundary of the 'cloud' $U$ of available controls, where the point of tangency is indicated as a red dot. Furthermore, in favour of computation efficiency in the algorithm, $H$ minimisation is cascaded to the individual wheel level, with the assumption that between each sample time the load transfer caused by braking and steering forces is small. More explanations are motivated in Yang et al. (2012a). It is worth noting that Hamiltonian minimisation makes full use of the non-linear tyre forces, and the linearised equations are only used to compute the co-states ratio; this limited use of the linearised model is thought to explain the high degree of correlation to the results seen in the nonlinear open-loop optimisations. 
Figure 1 Hamiltonian minimisation strategy. The cyan area represents the attainable $F_{y g}^{a c t} / \mathrm{m}$ and $M_{z}^{a c t} / I_{z z}$ subjected to non-linear tyre force limits. The red circle shows the optimal choice (see online version for colours)

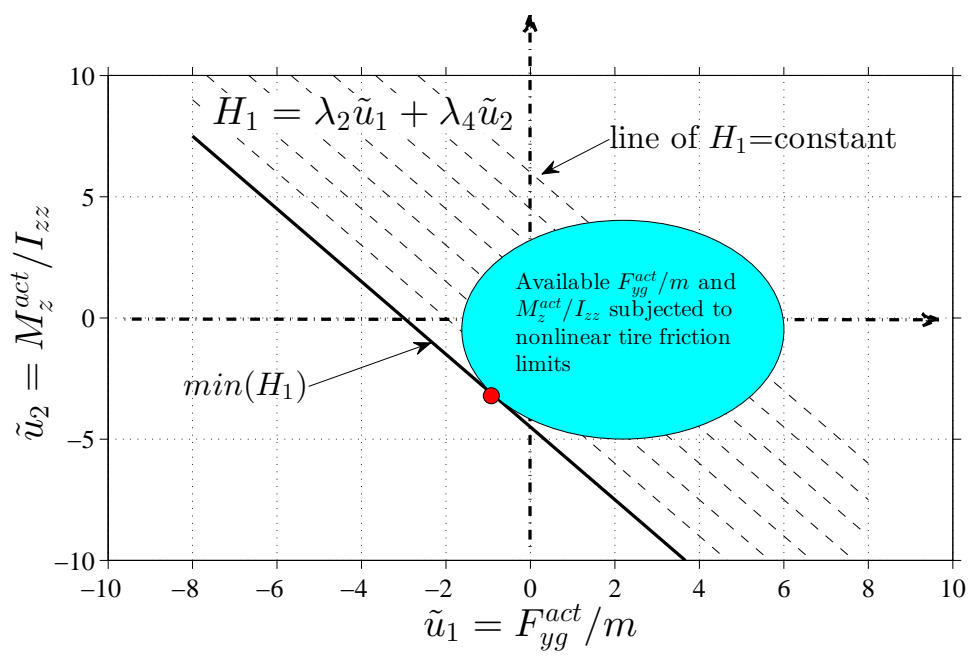

In the approximation assumed above, the co-state equations are linear with constant coefficients; thus, we have an explicit form of solution:

$$
\lambda(t)=\mathrm{e}^{-A^{\mathrm{T}}(t-t)} \cdot \lambda\left(t_{f}\right)
$$

where $\lambda_{f}=\lambda\left(t_{f}\right)=\left[Y_{f}, 0,0,0\right]^{\mathrm{T}}$. The solution depends on two parameters, $Y_{f}$ and $t_{f}$, but since the optimal control only depends on the co-state ratios, this leaves just one unknown parameter, $t_{f}$, to be determined. In Section 4, the new QLOC control is evaluated for two examples of post-impact dynamics, comparing results with numerical optimisations; in these two examples, $t_{f}$ was estimated from the numerical optimisation results, and the results are similar to those obtained with the estimation algorithm defined in Yang et al. (2012a).

A closed-loop form of the controlled system with the extended QLOC is shown in Figure 2. Here for the present paper, ideal sensor data and states estimators are assumed.

Figure 2 Block diagram of the closed-loop controlled system (see online version for colours)

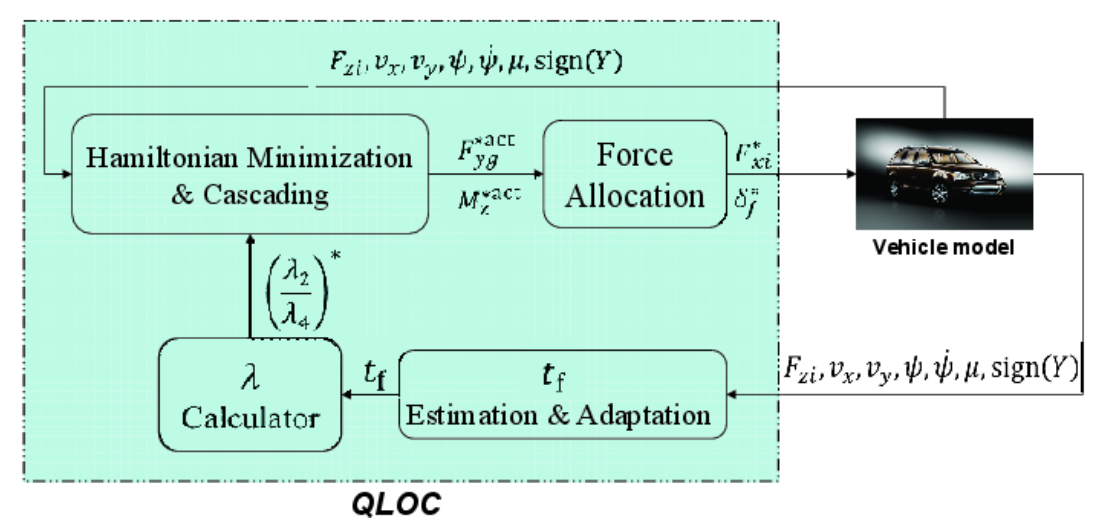




\section{Example cases - comparisons with open-loop optimisations}

Here simulation results are shown with the controller (QLOC) and numerical optimisation using JModelica.org (JM), which is a Modelica-based open source platform for optimisation, simulation and analysis of complex dynamic systems (Åkesson et al., 2010). The comparison in this section uses the 6-DOF model introduced in Section 2.1, for both the closed-loop controlled system and open-loop numerical optimisations.

We consider two example post-impact conditions. These cases are shown in Table 1. Case 1 involves yaw and lateral motions which are both disturbed to higher amplitudes, after side impact from right in front of $\mathrm{CG}$; case 2 represents slightly disturbed yaw motions which are in opposite direction of post-impact side-slip angle, after a typical sideswipe behind CG. The post-impact initial vertical velocity, roll velocity, pitch velocity and steering angle are assumed zero in this paper. The time duration of impact $t_{\text {imp }}$ is assumed to be $150 \mathrm{~ms}$; with linear interpolation of yaw rate, the post-impact yaw angle in Table 1 is calculated as

$$
\psi_{\mathrm{PI}}=\dot{\psi}_{\mathrm{PI}} \cdot t_{\text {imp }} / 2
$$

Table 1 Post-impact initial conditions of example cases

\begin{tabular}{lcccc}
\hline Case & $\dot{\psi}_{\mathrm{PI}}[\% \mathrm{~s}]$ & $\psi_{\mathrm{PI}}\left[{ }^{\circ}\right]$ & $Y_{\max }^{[1]}[\mathrm{m}]$ & $Y_{\max }^{[2]}[\mathrm{m}]$ \\
\hline 1 & 115 & 8.6 & 3.65 & 5.25 \\
2 & -29 & -2.1 & 0.62 & 0.63 \\
\hline Notes: & $v_{\mathrm{PI}}=15 \mathrm{~m} / \mathrm{s}, \beta_{\mathrm{PI}}=15^{\circ}, t_{\text {imp }}=0.15 \mathrm{~s}, \mu=0.9 . Y_{\max }^{[1]}$ is with EPAS+FricBrk and \\
& $Y_{\max }^{[2]}$ is with FricBrk.
\end{tabular}

For case 1, the optimal path is shown in Figure 3. It shows the vehicle motion as well as the available and actual tyre forces during the post-impact event; the solid blue line represents the force magnitude and direction at each tyre, and the purple lines map out a sector of attainable tyre forces, assuming the individual braking forces were varied at the currently selected steering angle. The available tyre forces are aggregated to find the set of resultant force $F_{y g}$ and yaw moment $M_{z}$ available at any instant. Previous work in Yang et al. (2012c) showed that with only friction brakes, the optimal $Y_{\max }$ can be achieved by applying maximum $F_{y g}$ opposing $Y$; one consequence there is that with brakes only, the vehicle turned more than $90^{\circ}$ in order to minimise $Y_{\max }$ (see Figure $3 \mathrm{~b}$ ). Here, with the added freedom of steering by EPAS, the high initial yaw velocity can be suppressed sufficiently by correcting the yaw moment so that the vehicle keeps facing forward (see Figure 3a). As shown in Table 1, $Y_{\max }$ is reduced by about $30 \%$ thanks to the presence of EPAS.

The wheel-level actuation in terms of tyre braking forces and front axle steering angle are also shown in Figures 4 and 5. It can be seen that, for QLOC, at the first $0.4 \mathrm{~s}$, the right tyres are braked more than the left ones, giving a large correcting yaw moment. The steering signals show the interesting feature that maximum angle and ramp-up rate are commanded throughout (the finite slope being due to the rate limiter in the EPAS system model). This indicates that, for the post-impact kinematics of this example type, large 
steering angle helps locate the available tyre force sectors, especially those at front axle, at a suitable angle within the friction circle so as to attain the best available $M_{z}^{\text {act }}$ and $F_{y g}^{a c t}$. The availability of these two critical vehicle-level control inputs over time is further analysed in the following. When compared to the numerical optimisation (JM), steer and most brake requests are largely similar, while it can be noticed that front right tyre is braked less by QLOC. (Differences on rear right tyre are considered negligible because of the very low vertical load on it.) JM requested more braking on the front right tyre that has generated more correcting yaw moment. As is known, braking will reduce the lateral grip and thus global lateral forces; thus, QLOC in this case prioritised the lateral force so as to instantaneously reduce $Y$, rather than applying brake forces even if they would reduce vehicle speed as well as yaw motion subsequently. Figure 6 shows that these slight adjustments in control modes actually make QLOC slightly better than JM in terms of reducing $Y_{\max }$.

Figure 3 Case1: optimal path and tyre force vectors using QLOC (see online version for colours)

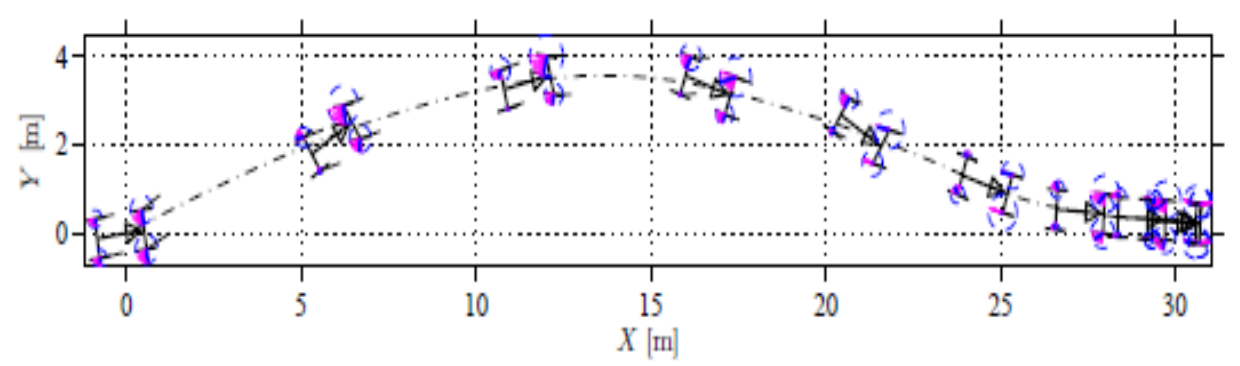

(a) The time interval between car plots: $0.45 \mathrm{~s}$

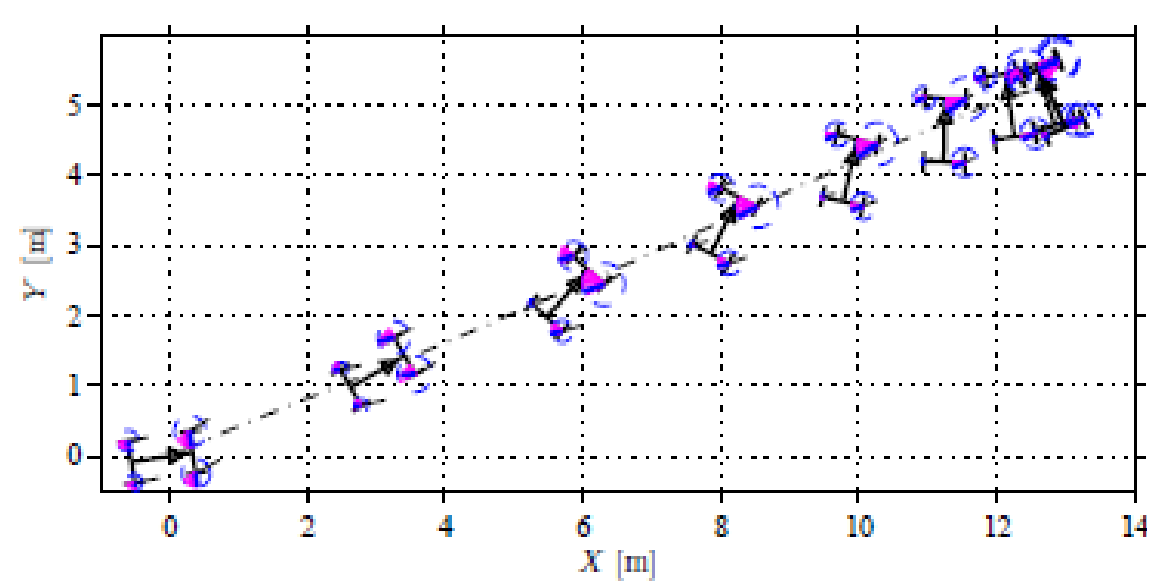

(b) The time interval between car plots: $0.23 \mathrm{~s}$. Brake-only, with zero road wheel steer angle 
Figure 4 Case 1: braking forces at tyres (normalised), until $Y_{\max }$, using QLOC with a 6-DOF vehicle model, compared to numerical optimisation (JM) (see online version for colours)
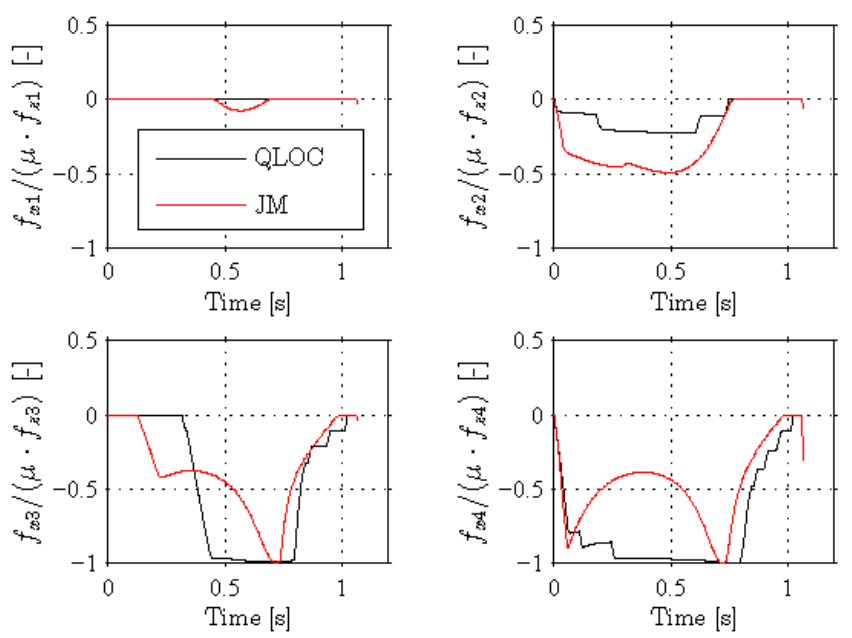

Figure 5 Case1: steering angle at front axle, until $Y_{\max }$, using QLOC with a 6-DOF vehicle model, compared to numerical optimisation (JM) (see online version for colours)

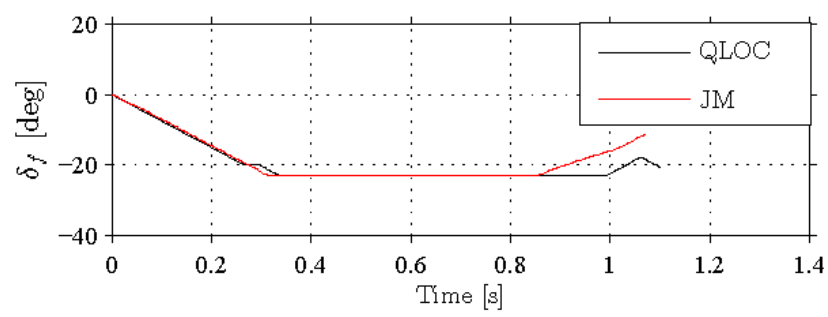

Figure 6 Case 1: closed-loop path controller (QLOC) vs. open-loop numerical optimisation (JM). Simulations for JM are completed at $Y_{\max }\left(t_{f}=1.1 \mathrm{~s}\right)$, where settling control is switched on. Red vertical line shows the switching instant (see online version for colours)
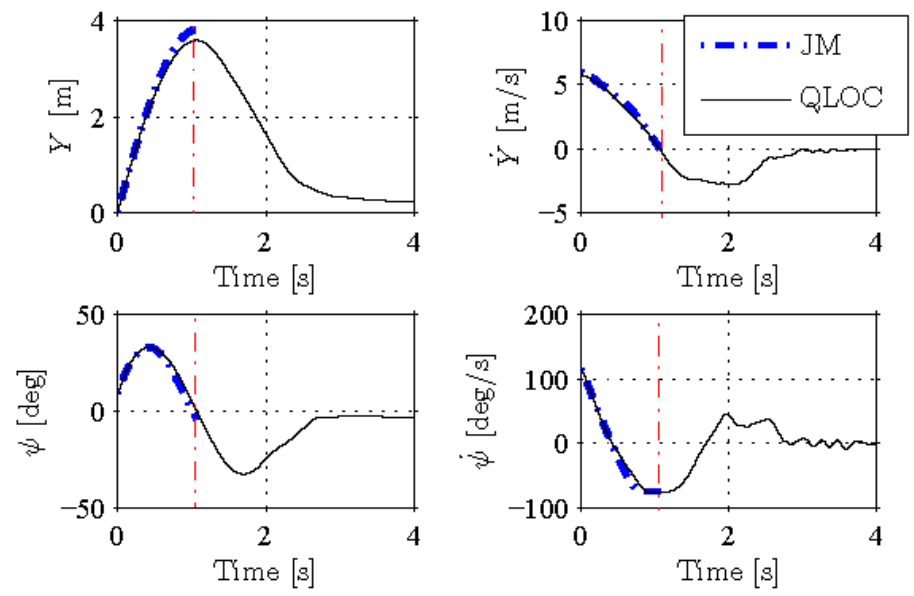
Figure 6 shows the time histories of the four state variables for case 1, together with comparison results from independent open-loop non-linear optimisations. For closedloop control, the complete control performance including the settling phase is shown. The settling controller is not the subject of the current paper (see Yang et al., 2012b). In this example, the post-impact initial yaw velocity is actually outside the range of effective operation for brake-only QLOC (see Yang et al., 2012a), while it is within the effective region of QLOC when the EPAS actuator is added (see Yang et al., 2012c). The results closely match with that from numerical optimisation, indicating that QLOC controller is extended properly to reach the optimum.

Looking into the control input $\tilde{\mathbf{u}}$, the attainable active force $F_{y g}^{\text {act }}$ and yaw moment $M_{z}^{a c t}$ are obtained, after excluding the contributions from tyre lateral force at zero braking forces and zero steering angles. For case 1, they are shown as the black 'cloud' (scatter plot) in the 2D plane (see Figure 7). These snapshots of available forces and moments are shown with equal spacing between $t=0$ and $t=t_{f}$, before $Y_{\max }$ is reached. The red line indicates the line of minimum Hamiltonian which determines the optimal balance between $F_{y g}^{a c t}$ and $M_{z}^{\text {act }}$ via the red dot which represents the choice of force and moment obtained by QLOC. It can be seen that the optimal control stays at the boundary of the brute-force 'cloud' in spite of the simplified algorithm for Hamiltonian minimisation. The optimal balance evolves from an initial $M_{z}^{a c t}$-bias to $F_{y g}^{a c t}$-bias. It is also noted that from $t=0.32 \mathrm{~s}$ to $t=0.42 \mathrm{~s}$, the red dot jumps upwards directly to the leftmost point of cloud. This is not surprising if one notices the shape of the cloud's left boundary, which is rather flatter than the brake-only clouds shown in Yang et al. (2012a) and Yang et al. (2012b). The lower left point of active clouds migrates rapidly from $(0,0)$ to $(-20,-20)$, which helps the path control problem here. This is due to the rapid initial changes in vehicle states, which in turn define the availability of active forces and moments. Yaw moment plays a critical role in changing the vehicle states in a beneficial direction - hence, $F_{y g}^{a c t}$ is not instantaneously minimised. As mentioned above, the inclusion of a steering actuator increases the available correcting yaw moment in comparison with using brake actuators alone. For a comprehensive comparison of the optimal control sequences at various post-impact kinematics, please refer to Yang et al. (2012c).

For case 2, the vehicle path is shown in Figure 8 and the comparison with numerical optimisation is shown in Figure 9. Similar to case 1, the two independent approaches show almost identical results. Figure 10 shows the available forces and moments, where the line of minimum $H$ starts with a very high gradient; taken together with the shape of clouds, the optimal choice is roughly equivalent to minimising the instantaneous value of $F_{y g}$, with minimal regard for $M_{z}$, i.e. control degenerates to the above-mentioned $F_{y g}$ control. It is also noted that the red dots stay near $(0,0)$, which indicates that the application of braking and steering controls will not improve the post-impact response ( $Y_{\max }$ reduction). This aspect is also reflected in Table 1, i.e. added steering authority hardly influences $Y_{\max }$. 
Figure 7 Case 1: available active global forces $F_{y g}^{a c t}$ and moments $M_{z}^{a c t}$, until $Y_{\max }$, using QLOC (see online version for colours)
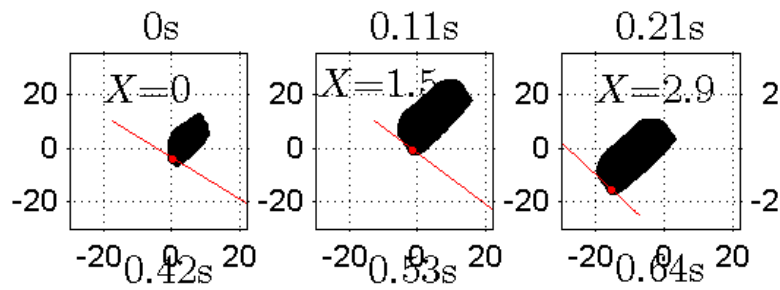

$0.32 \mathrm{~s}$
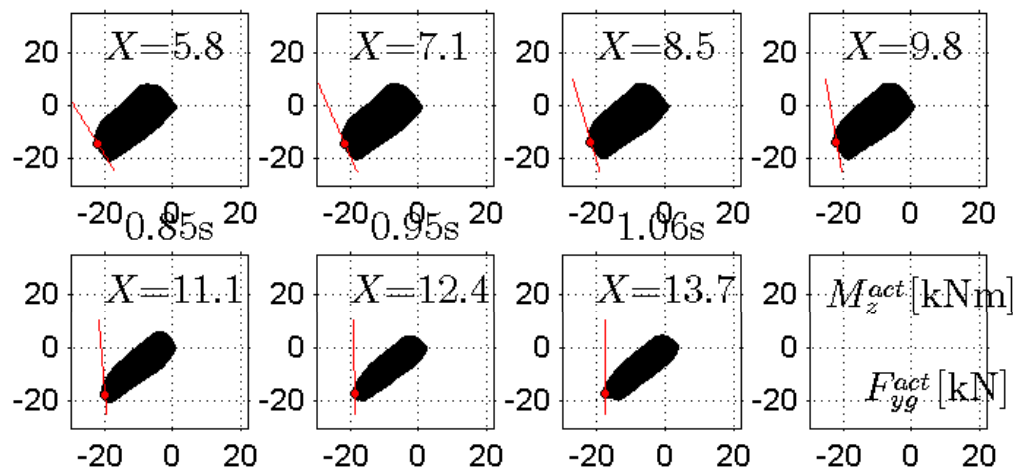

Figure 8 Case 2: optimal path and tyre force vectors using QLOC. The time interval between car plots: $0.25 \mathrm{~s}$ (see online version for colours)

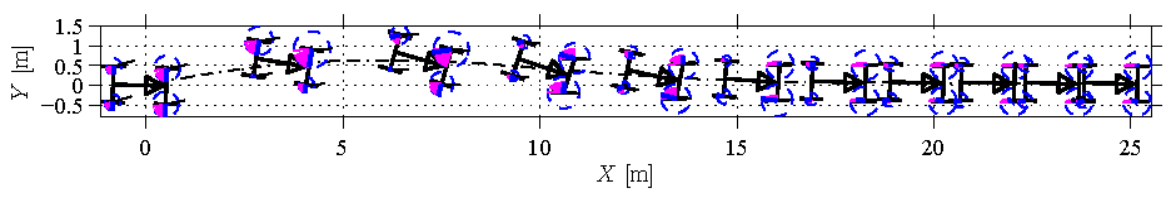

Figure 9 Case 2: closed-loop path controller (QLOC) vs. open-loop numerical optimisation (JM). Simulations for JM are completed at $Y_{\max }\left(t_{f}=0.35 \mathrm{~s}\right)$, where settling control is switched on. Red vertical line shows the switching instant (see online version for colours)
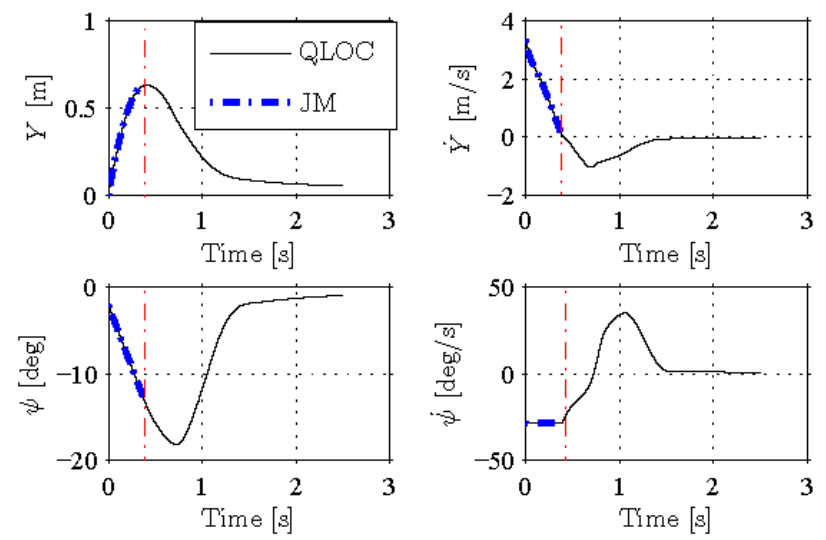
Figure 10 Case 2: available active global forces $F_{y g}^{\text {act }}$ and moments $M_{z}^{\text {act }}$, until $Y_{\max }$, using QLOC (see online version for colours)

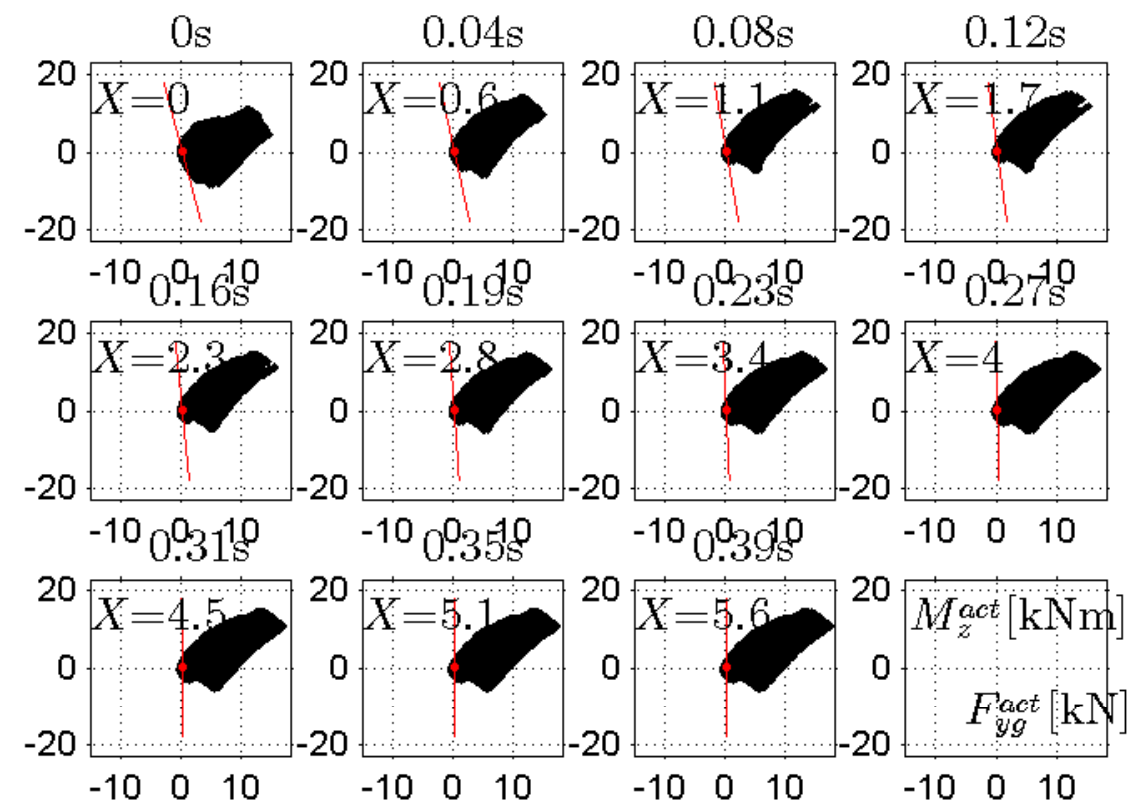

\section{Simplified algorithm for real-time control}

In real-time tests using high-fidelity vehicle simulation models and actual vehicles, it is expected that control allocation using the brute-force method above will be a computational bottleneck. Hence, the control allocation part of the algorithm is now simplified by directly using actuator rate limits when calculating the available vehicle forces and moments. As above, the results are compared with open-loop numerical optimisations which respect the same actuator limits during optimisation.

Here both saturation and rate limits are included in the allocation algorithm; available tyre brake forces and steer angles are expressed in three modes, i.e. decrease, hold and increase. For brake control at individual wheels, the set of available friction brake forces $F_{x w i}^{a l b}$ is based on the optimal force request $F_{x w i}^{*}$ at the previous sample time:

$$
F_{x w i}^{a l b}(k+1)= \begin{cases}-\operatorname{sgn}\left(v_{x w i}\right) \cdot\left[0,\left|F_{x w i}^{*}(k)\right|,\left|F_{x w i}^{*}(k)\right|+\Delta_{1}\right], & \left|F_{x w i}^{*}(k)\right|+\Delta_{2}<0 \\ -\operatorname{sgn}\left(v_{x w i}\right) \cdot\left[\left|F_{x w i}^{*}(k)\right|+\Delta_{2},\left|F_{x w i}^{*}(k)\right|,\left|F_{x w i}^{*}(k)\right|+\Delta_{1}\right],\left|F_{x w i}^{*}(k)\right|+\Delta_{2} \geq 0\end{cases}
$$

where $\Delta_{1}=\Delta_{b 1} \cdot t_{s}, \Delta_{2}=\Delta_{b 2} \cdot t_{s}, k$ denotes the current sample, $t_{s}$ is the sampling time, $\Delta_{b 1}$ is the rising rate limit of requested brake force and $\Delta_{b 2}$ is the falling rate limit. It is considered that the brake forces are saturated by the limited road friction (see equation 3 ), rather than by the maximum power of the hydraulic brake system. See Appendix A for values of the actuators' parameters. 
For EPAS, the front wheel steer angle is constrained to be within $\delta_{\text {lim }}= \pm 0.4 \mathrm{rad}$, and subject to the rate limit $\Delta_{b}$; then, the available steer angles at the subsequent sample time are contained in the set:

$$
\delta_{f}^{a l b}(k+1)=\left[\delta_{f}^{*}(k)-\Delta_{\delta} \cdot t_{s}, \delta_{f}^{*}(k), \delta_{f}^{*}(k)+\Delta_{\delta} \cdot t_{s}\right]
$$

Figure 11 shows that the performance of QLOC does not deteriorate due to the simplification of the control allocation algorithm. This is not surprising if one notices the patterns of actuators mode shown in Figures 4 and 5; it can be observed that at most time instants, requested steering and braking are either unchanged or drastically changed between consecutive sample times. That implies the available tyre forces are largely constrained by the rate limit of actuators; this is also consistent with the fact that for case 1 here, $Y_{\max }$ can be further reduced if actuators control is no longer subject to rate limits. More case studies can be conducted in the future to test this conclusion across the entire set of post-impact kinematics. Regarding the computation time comparison, it was estimated on a personal computer that the simplified algorithm takes about $15 \%$ of the CPU time used by the original one.

Figure 11 Case 1: closed-loop path controller (simplified QLOC considering actuator limits) vs. open-loop numerical optimisation (JM). Sampling time is $5 \mathrm{~ms}$
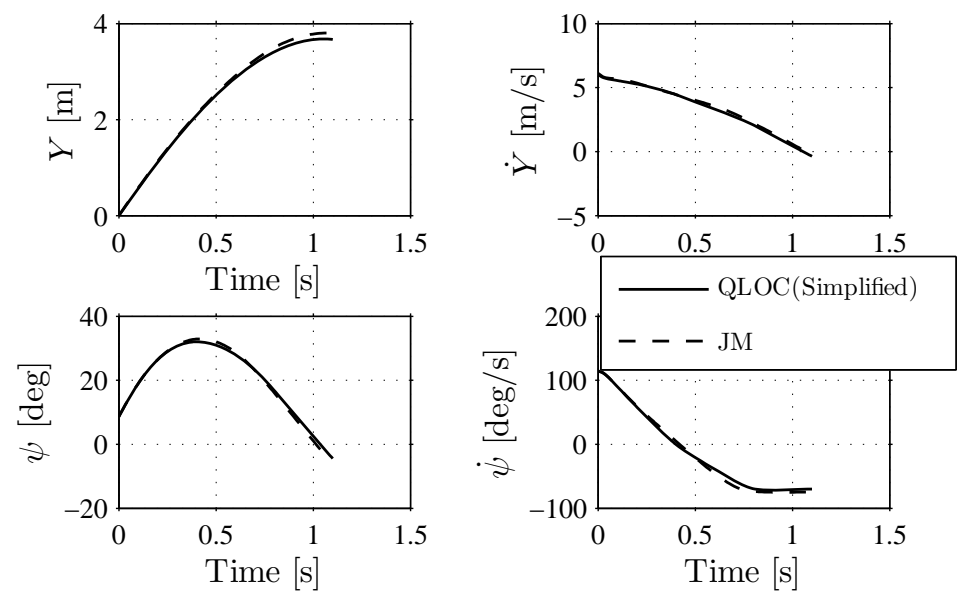

\section{Robustness test on a vehicle model for limit handling}

Until now the controller, designed around a 6-DOF vehicle model, was evaluated using the same model. This is appropriate when testing for optimality, but since the validation model is well matched to the controller, we cannot conclude that the controller would be effective in practice. It is therefore necessary to further test the controller using an experimental vehicle or - as an intermediate step - using a vehicle model which has additional non-linearities and additional degrees of freedom, and where only a subset of parameters are known to the controller. Hence, before actual vehicle implementation is considered, the robustness with respect to vehicle and tyre properties is now tested, i.e. a higher fidelity vehicle model is used to evaluate the sensitivity of control performance to model uncertainties. 
Here a multi-body dynamics vehicle model is utilised which was validated at different steady-state and transient driving situations, especially in limit handling manoeuvres. This model was developed in Dymola $/$ Vehicle Dynamics Library, and parameterised according to an on-market SUV model (Andreasson and Jonasson, 2008). It includes increased detail of the various subsystems of an SUV vehicle chassis system: there is 6 DOF for each suspension linkage that accounts for compliances, 1 DOF for wheel travel and 6 DOF for body motion, plus 1 rotational DOF around each wheel shaft and 2 DOF for transient response at each tyre; in total, this model has $(6+1+1+2) \times 4$ $+6=46$ degrees of freedom; more information about the model and its validation can be found in Jonasson et al. (2008). Since the focus of the robustness tests in the present paper is in relation to model complexity and uncertainty, ideal sensor data and states estimators are still assumed. As a benchmark, open-loop numerical optimisations are rerun using vehicle data more appropriate to the SUV vehicle mentioned. However, one should bear in mind that in solving the non-linear programming problem within an optimisation scheme, model complexity is highly limited; therefore, the 6-DOF model of Section 2.1 is retained for numerical optimisation, with the vehicle parameters modified according to the SUV model. Hence, the open-loop optimisations are somewhat idealised in terms of what can actually be achieved when controlling the high-order SUV model.

Figure 12 Case 1: closed-loop path controller (QLOC with a high-fidelity vehicle model) vs. open-loop numerical optimisation (JM) (see online version for colours)
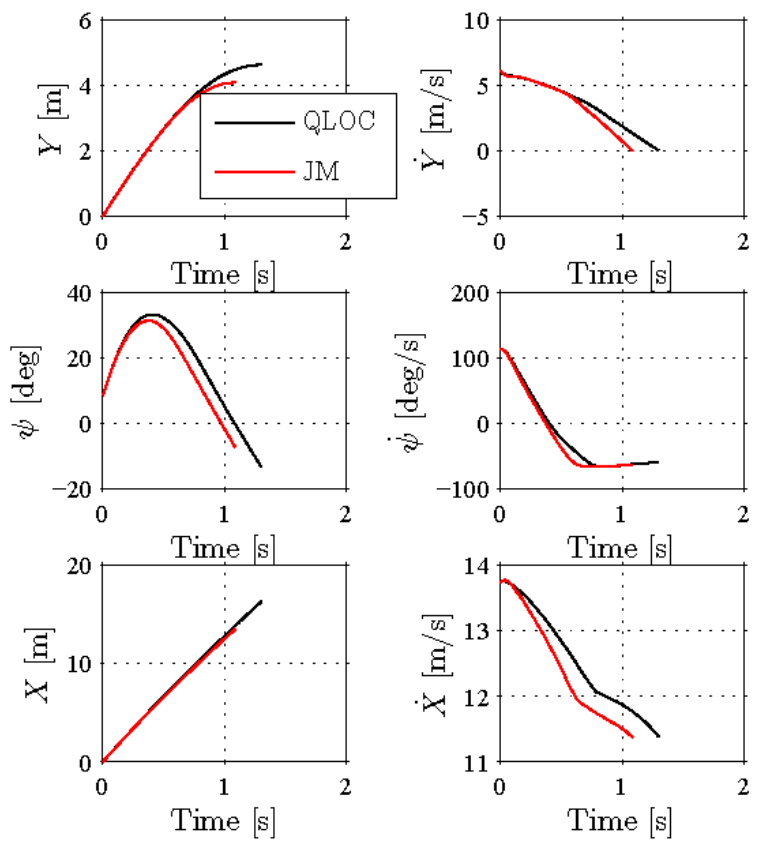

Figure 12 shows the state variables as compared to JM open-loop optimisation. As expected, the state trajectories using QLOC are not as close to JM as in Figure 6. It can be seen that from the very beginning, control on $X$-dynamics is noticeably different between the two methods. This is understood when the tyre forces are compared; for the validated SUV model, a full version of the Pacejka tyre model is used, and this has 
considerably less lateral stiffness compared with the longitudinal stiffness. This implies that at combined slip situation, the tyre friction circle is anisotropic, unlike in the simplified tyre model assumed by the controller. It is thus seen in Figure 13 that the tyre force vector does not reach the border of an isotropic friction circle (see the zoom-in view of one plotted vehicle in the figure); it is thus expected that the 46-DOF model is more challenging to control than the 6-DOF model used by JM.

Figure 13 Case 1: optimal path and tyre force vectors, using QLOC with a high-fidelity vehicle model. The time interval between car plots: $0.13 \mathrm{~s}$. The zoom-in figure shows the individual tyre forces in greater detail, and specifically that lateral force does not reach the friction circle at the front left wheel (see online version for colours)



Furthermore, brake forces are shown in Figure 14; similar to Figure 4 where a 6-DOF model was used, controls overlapped with each other mostly, except for the front right wheel. The comparison of front axle steering angle is shown in Figure 15. Similar to before, both methods requested maximum angle and ramp-up rate; while for QLOC with the 46-DOF SUV model here, large steer angle is requested for a longer period of time until $Y_{\max }$ is reached, in order to compensate the lack of lateral grip.

Figure 14 Case 1: braking forces at tyres (normalised), using QLOC with a high-fidelity vehicle model, compared to numerical optimisation (JM) (see online version for colours)
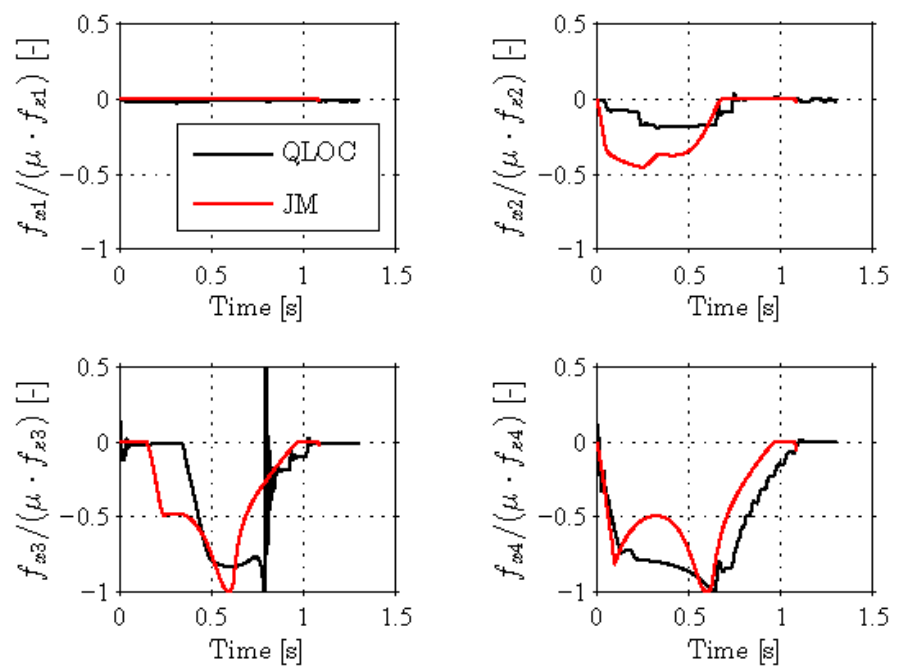
Figure 15 Case 1: steering angle at front axle, using QLOC with a high-fidelity vehicle model, compared to numerical optimisation (JM) (see online version for colours)

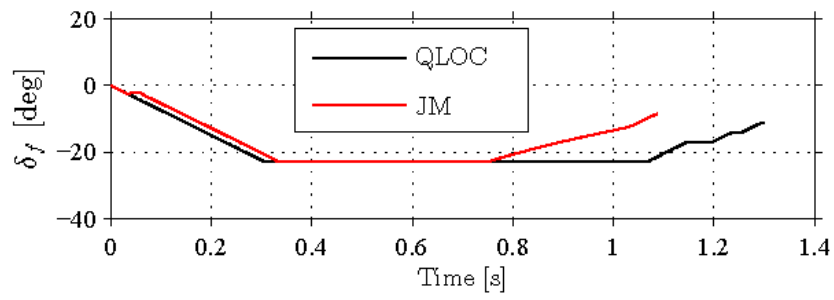

Figure 16 shows the available active forces and moments, i.e. 'cloud', computed by QLOC that uses the simplified Pacejka tyre model shown in equation (2). The red circle is computed from the instantaneous tyre forces of the SUV vehicle. The slope of straight Hamiltonian lines remains to be the co-states ratio computed by the reduced and linearised model in Section 2.2. It shows that during roughly the first $0.6 \mathrm{~s}$, the optimal choice stays at the boundary of 'cloud'. This suggests that the effects of model differences do not strongly influence control system optimality during this period. But as time goes by, the model error in the controller seems to accumulate so that the red circle appears inside the 'cloud'. The apparent sub-optimality at the zero time instant can be discounted - it is largely due to the initialisation of the SUV model in Dymola ${ }^{\circledR}$; since the post-impact kinematics are not generated by a vehicle-to-vehicle collision model, a limited number of states can be adequately estimated and assigned directly to the vehicle model. Therefore, it takes a short time for the model to fully develop a fully trimmed set of PI states; this particularly affects the tyre forces during the instants immediately after the impact.

Figure 16 Case 1: available active global forces $F_{y g}^{a c t}$ and moments $M_{z}^{a c t}$, until $Y_{\max }$, using QLOC with a high-fidelity vehicle model (see online version for colours)

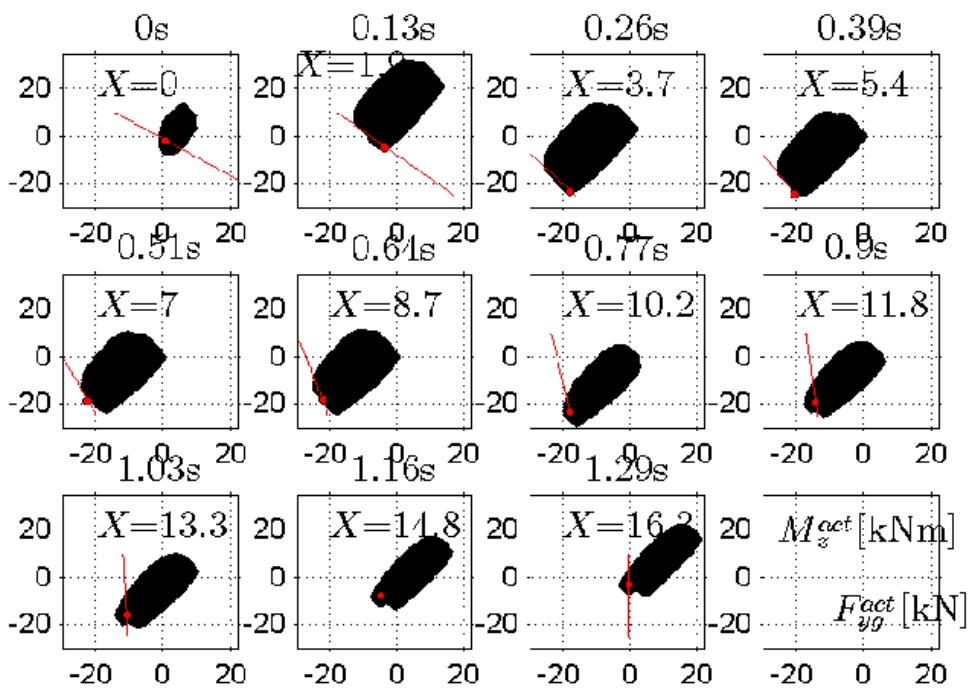


Similar tests were conducted with several other cases having quite different post-impact kinematics. Overall the controller has shown highly satisfactory performance in regulating the path lateral deviation of the high-fidelity vehicle model. To improve the optimality further, additional algorithms can be implemented, including an estimator of the tyre model parameters, e.g. road friction and cornering stiffness, so as to be adaptive and reduce the overall level of model uncertainty. It is also expected that a lower level wheel slip controller can be used to improve performance; this will help to generate more precise brake force requests which would indeed minimise the Hamiltonian as calculated by vehicle-level QLOC controller.

\section{Discussions and conclusions}

In this paper, we have further evaluated the QLOC developed in previous work, including a steering actuator to allow further control system integration for post-impact manoeuvres. The inclusion of the steering actuator does not change the underlying QLOC methodology, and our extension to the algorithm has been implemented without further tuning or correction. Comparison with independent results from non-linear numerical optimisation indicates that QLOC can deal seamlessly with a variety of different actuator configurations. This property further suggests that QLOC may be made robust to actuator faults, provided that fault detection and isolation systems are used to determine any failure states of the actuators; it should not require any fundamental change in the core control architecture.

Simulations using the proposed closed-loop QLOC control have verified the optimality compared to open-loop numerical optimisations. Two distinctive cases were analysed, each showing a different use of vehicle-level forces and moments. Even though QLOC uses a linear vehicle model for estimating co-state ratios, it makes full use of the tyre friction limits and this simplification does not appear to reduce the effectiveness of the controller.

As expected, the availability of correcting yaw moments is enhanced by the added steering actuator. This augmentation of control authority makes it possible to attain more favourable global lateral forces within the future time horizon; in one case, this is found to substantially reduce $Y_{\max }$, while in the other case hardly any improvement resulted. These differences were investigated previously in a comprehensive way - using purely open-loop control optimisation - and the effective region of added steer actuator was identified in Yang et al. (2012c).

In this work, a 6-DOF vehicle model has been used for controller development and initial validation, as compared to the 3-DOF model used previously. It has been verified that optimality is not degraded because of this - for example, roll and pitch dynamics are fully included in both the numerical optimisation and the control system evaluation. The control algorithm has been challenged further by applying the control to another independent multi-body high-CG vehicle model; results of example cases have shown that the controller performance is insensitive to the exact choice of plant model. For further realism, considering efficient evaluation in real time, the control allocation part in the algorithm was simplified by incorporating the actuator dynamics; it shows the computation time can be greatly reduced while losing nothing by way of closed-loop performance. 
In the selected examples, a complete control time history was provided, including the vehicle settling phase. It is expected that in future work, the settling controller should be improved in order to better track a desired profile of lateral speed in road coordinates. Inclusion of the steering actuator may actually increase the risk of overshooting after the point of maximum lateral displacement is reached, which may impose a more challenging task for the settling phase.

As stated in the Introduction, more research should be done to evaluate the humanmachine interface of such control features, prior to the implementation into production vehicle. For instance, using the EPAS system, an abrupt steering torque input superimposed onto the hand wheel may even injure the driver's hands. Hence, it may be necessary to limit the rotational torque applied to the steering wheel, even if this degrades the overall performance of the post-impact control system. It is also critical to determine the timing and actions associated with handing control back to the driver, especially to avoid misunderstandings between the vehicle controller and human driver.

During the initial impact, some damage on the vehicle may be sustained to cause sensors or actuators to fail. It is expected that sensors can be upgraded to sustain relatively high accelerations and velocities under the circumstances of an external impact. Provided faults or partial system failures can be identified in real-time, control adaptation should be feasible. In the case of actuator faults, adaptive fault-tolerant control may be needed to continuously update the estimation of constraints on Hamiltonian minimisation. Similar to the identification of the external scenario, the topic of internal system fault detection, identification and compensation is worthy of much deeper consideration in the future.

Future work may be required to include a vehicle-to-vehicle collision model, so that the initial impact can be more thoroughly emulated. This will provide more complete information about vehicle kinematics immediately after the collision, for instance the post-impact steering angle. State and parameter estimators will also be important for further evaluating the robustness of the post-impact controller, as appropriate to high degree of freedom vehicle simulations and to actual vehicle tests.

\section{Acknowledgements}

This work was in part financed by Swedish Governmental Agency for Innovation Systems (VINNOVA) and associated with Vehicle and Traffic Safety Centre (SAFER). The authors are grateful to Ivar Torstensson at Modelon AB for the support to work with the multi-body Modelica model.

\section{References}

Åkesson, J., Årzén, K-E., Gäfvert, M., Bergdahl, T. and Tummescheit, H. (2010) 'Modeling and optimization with optimica and jmodelica.org-languages and tools for solving large-scale dynamic optimization problem', Computers and Chemical Engineering, Vol. 34, No. 11, pp.1737-1749.

Andreasson, J. and Jonasson, M. (2008) 'Vehicle model for limit handling: implementation and validation', in Proceedings of the $6^{\text {th }}$ Modelica Conference, University of Applied Sciences, Bielefeld, Germany. 
Bryson Jr., A.E. and Ho, Y-C. (1975) Applied Optimal Control: Optimization, Estimation and Control, revised ed., Taylor \& Francis, Abingdon, UK.

Fay, P., Sferco, R. and Richard, F. (2001) 'Multiple impact crashes - consequences for occupant protection measures', Proceedings of the 2001 IRCOBI Conference on the Biomechanics of Impact, Isle of Man, UK.

Häussler, A., Schäffler, R., Georgi, A. and Stabrey, S. (2012.) 'Networking of airbag and ESP for prevention of further collisions', ATZ - Automobiltechnische Zeitschrift, Vol. 114, pp.22-26.

Jonasson, M., Andreasson, J., Jacobson, B., Stensson Trigell, A. (2008) 'Modelling and parameterisation of a vehicle for validity under limit handling', Proceedings of 10th International Symposium on Advanced Vehicle Control, Kobe, Japan.

Jonasson, M., Andreasson, J., Jacobson, B. and Trigell, A.S. (2010) 'Global force potential of overactuated vehicles', Vehicle System Dynamics, Vol. 48, No. 9, pp.983-998.

Kusachov, A. and Mouatamid, F.A. (2012) Post Impact Braking Verification in Motion Platform Simulator, Master's Thesis, Chalmers University of Technology, Gothenburg, Sweden.

Pacejka, H.B. (2006) Tire and Vehicle Dynamics, 2nd ed., Society of Automotive Engineers, Inc, Warrendale, PA.

Sander, U., Mroz, K., Boström, O. and Fredriksson, R. (2009) 'The effect of pre-pretensioning in Multiple Impact Crashes', Proceedings of the 21st (Esv) International Technical Conference on the Enhanced Safety of Vehicles, Stuttgart, Germany.

Sundström, P., Jonasson, M., Andreasson, J., Stensson Trigell, A. and Jacobson, B. (2010) 'Path and control optimization for over-actuated vehicles in two safety-critical maneuvers', Proceedings of the 10th International Symposium on Advanced Vehicle Control, Loughborough, UK.

Yang, D., Jacobson, B. and Lidberg, M. (2009) 'Benefit prediction of passenger car post impact stability control based on accident statistics and vehicle dynamics simulations', Proceedings of the. 21st International Symposium on Dynamics of Vehicles on Roads and Tracks', Stockholm, Sweden.

Yang, D., Gordon, T.J., Jacobson, B., Jonasson, M. and Lidberg, M. (2011) 'Optimized brakebased control of path lateral deviation for mitigation of secondary collisions', Proceedings of the Institution of Mechanical Engineers, Part D: Journal of Automobile Engineering, Vol. 225, No. 12, pp.1587-1604.

Yang, D., Gordon, T.J., Jacobson, B. and Jonasson, M. (2012a) 'Quasi-linear optimal path controller applied to post impact vehicle dynamics', IEEE Transactions on Intelligent Transportation Systems, Vol. 13, No. 4, pp.1586-1598.

Yang, D., Gordon, T., Jacobson, B. and Jonasson, M. (2012b) 'A nonlinear post-impact path controller based on optimized brake sequences', Vehicle System Dynamics: International Journal of Vehicle Mechanics and Mobility, Vol. 50 (supplement), pp.131-149.

Yang, D. Jacobson, B., Jonasson, M. and Gordon, T. (2012c) 'Minimizing vehicle post-impact path lateral deviation using optimized braking and steering sequences', Proceedings of the 11th International Symposium on Advanced Vehicle Control (AVEC '12), Seoul, Korea.

Zhou, J., Lu, J.and Peng, H. (2010) 'Vehicle stabilisation in response to exogenous impulsive disturbances to the vehicle body', International Journal of Vehicle Autonomous Systems, Vol. 8, Nos. 2-4, pp.242-262. 


\section{Appendix A Nomenclature and parameters}

Table A1 Nomenclature I

\begin{tabular}{|c|c|c|c|}
\hline Notation & Value & Unit & Description \\
\hline$B_{1}, B_{2}$ & {$[19.2]$} & {$[\mathrm{N} / \mathrm{rad}]$} & Front tyre cornering stiffness \\
\hline$B_{3}, B_{4}$ & {$[21.3]$} & {$[\mathrm{N} / \mathrm{rad}]$} & Rear tyre cornering stiffness \\
\hline$C_{\mathrm{i}}$ & {$[1]$} & {$[-]$} & Shape factor of tyre model \\
\hline$C_{\alpha_{f}}$ & {$[13,000]$} & {$[\mathrm{N} / \mathrm{rad}]$} & Front cornering stiffness in controller model \\
\hline$C_{\alpha_{r}}$ & {$[7800]$} & {$[\mathrm{N} / \mathrm{rad}]$} & Rear cornering stiffness in controller model \\
\hline$F_{w \mathrm{i}}$ & {$[-]$} & {$[\mathrm{N}]$} & Brake pad force on wheel i \\
\hline$F_{x w i}$ & {$[-]$} & {$[\mathrm{N}]$} & $\begin{array}{l}\text { Requested brake force, transformed from brake pad } \\
\text { force onto tyre } \mathrm{i}\end{array}$ \\
\hline$F_{x i}$ & {$[-]$} & {$[\mathrm{N}]$} & Longitudinal tyre force on wheel i \\
\hline$F_{x g}$ & {$[-]$} & {$[\mathrm{N}]$} & $\begin{array}{l}\text { Aggregated longitudinal force on body, in road } \\
\text { coordinate }\end{array}$ \\
\hline$F_{y i}$ & {$[-]$} & {$[\mathrm{N}]$} & Lateral tyre force on wheel i \\
\hline$F_{y g}$ & {$[-]$} & {$[\mathrm{N}]$} & Aggregated lateral force on body, in road coordinate \\
\hline$F_{z i}$ & {$[-]$} & {$[\mathrm{N}]$} & Vertical tyre force on wheel i \\
\hline$F_{z g}$ & {$[-]$} & {$[\mathrm{N}]$} & Aggregated vertical force on body, in road coordinate \\
\hline$I_{x x}$ & 764.5 & {$\left[\mathrm{~kg} \mathrm{~m}^{2}\right]$} & Principal roll moment of inertia \\
\hline$I_{y y}$ & 3477 & {$\left[\mathrm{~kg} \mathrm{~m}^{2}\right]$} & Principal pitch moment of inertia \\
\hline$I_{z z}$ & 3258 & $\mathrm{~kg} \mathrm{~m}^{2}$ & Principal yaw moment of inertia \\
\hline$L_{y i}$ & {$[0.15]$} & {$[\mathrm{m}]$} & Tyre lateral relaxation length \\
\hline$X$ & {$[-]$} & {$[\mathrm{m}]$} & Vehicle longitudinal displacement in road coordinate \\
\hline$Y$ & {$[-]$} & {$[\mathrm{m}]$} & Vehicle lateral displacement in road coordinate \\
\hline$Z$ & {$[-]$} & {$[\mathrm{m}]$} & Vehicle vertical displacement in road coordinate \\
\hline$c_{12}$ & 20,006 & {$[\mathrm{~N} / \mathrm{m}]$} & Stabiliser stiffness, front \\
\hline$c_{34}$ & 16,088 & {$[\mathrm{~N} / \mathrm{m}]$} & Stabiliser stiffness, rear \\
\hline$d_{1}, d_{2}$ & 4500 & {$[\mathrm{Ns} / \mathrm{m}]$} & Damping coefficient, front \\
\hline$d_{3}, d_{4}$ & 3500 & {$[\mathrm{Ns} / \mathrm{m}]$} & Damping coefficient, rear \\
\hline G & 9.81 & {$\left[\mathrm{~m} / \mathrm{s}^{2}\right]$} & Gravitational acceleration \\
\hline$h_{c g}$ & 0.506 & {$[\mathrm{~m}]$} & Vehicle CG height over ground \\
\hline$h_{p}$ & 0.3 & {$[\mathrm{~m}]$} & Vehicle CG position forward pitch centre \\
\hline$h_{r}$ & 0.44 & {$[\mathrm{~m}]$} & Vehicle CG position over roll centre \\
\hline$i$ & $1,2,3,4$ & {$[-]$} & $\begin{array}{l}\text { 1: front left tyre, } 2 \text { : front right tyre, } 3 \text { : rear left tyre, } 4 \text { : } \\
\text { rear right tyre }\end{array}$ \\
\hline$k_{1}, k_{2}$ & 33,000 & {$[\mathrm{~N} / \mathrm{m}]$} & Spring stiffness, front \\
\hline$K_{3}, k_{4}$ & 56,000 & {$[\mathrm{~N} / \mathrm{m}]$} & Spring stiffness, rear \\
\hline
\end{tabular}


Table A2 Nomenclature II

\begin{tabular}{cccl}
\hline Notation & Value & Unit & Description \\
\hline$l_{f}$ & 1.033 & {$[\mathrm{~m}]$} & CG to front axle \\
$l_{r}$ & 1.682 & {$[\mathrm{~m}]$} & CG to rear axle \\
$m$ & 1625 & {$[\mathrm{~kg}]$} & Vehicle mass \\
$v_{P I}$ & {$[-]$} & {$[\mathrm{m} / \mathrm{s}]$} & Vehicle speed at the end of the first impact \\
$v_{x}$ & {$[-]$} & {$[\mathrm{m} / \mathrm{s}]$} & Longitudinal velocity in vehicle coordinate \\
$v_{x w i}$ & {$[-]$} & {$[\mathrm{m} / \mathrm{s}]$} & Longitudinal velocity of the wheel hub centre at each wheel $i$ \\
$v_{y}$ & {$[-]$} & {$[\mathrm{m} / \mathrm{s}]$} & Lateral velocity in vehicle coordinate \\
$w$ & 1.56 & {$[\mathrm{~m}]$} & Track width \\
$\alpha_{f}$ & {$[-]$} & {$[\mathrm{rad}]$} & Front axle side-slip angle \\
$\alpha_{r}$ & {$[-]$} & {$[\mathrm{rad}]$} & Rear axle side-slip angle \\
$\alpha_{i}$ & {$[-]$} & {$[\mathrm{rad}]$} & Tyre side-slip angle \\
$\beta$ & {$[-]$} & {$[\mathrm{rad}]$} & Vehicle side-slip angle: arctan $\left(v_{y} /\left|v_{x}\right|\right)$ \\
$\psi$ & {$[-]$} & {$[\mathrm{rad}]$} & Vehicle yaw angle \\
$\phi$ & {$[-]$} & {$[\mathrm{rad}]$} & Vehicle roll angle \\
$\theta$ & {$[-]$} & {$[\mathrm{rad}]$} & Vehicle pitch angle \\
$\mu_{i}$ & {$[0.9]$} & {$[-]$} & Road friction coefficient at each tyre \\
$\Delta_{b 1}$ & {$[25,000]$} & {$[\mathrm{N} / \mathrm{s}]$} & Rising rate limit of requested brake forces \\
$\Delta_{b 2}$ & {$[-83,000]$} & {$[\mathrm{N} / \mathrm{s}]$} & Falling rate limit of requested brake forces \\
$\Delta_{\delta}$ & {$[1.3]$} & {$[\mathrm{rad} / \mathrm{s}]$} & Rate limit of steering actuator \\
$\delta_{\text {lim }}$ & {$[ \pm 0.4]$} & {$[\mathrm{rad}]$} & Saturation limit of $\delta_{f}$ \\
$\delta_{f}$ & {$[-]$} & {$[\mathrm{rad}]$} & Road wheel steer angle at front axle \\
$\lambda$ & {$[-]$} & {$[-]$} & Lagrange multiplier vector whose elements are called \\
& & & co-states in optimal control problem \\
\hline & & &
\end{tabular}

